\title{
Treatment of refractory thrombotic thrombocytopenic purpura with Caplacizumab : A case report
}

zakaria maat $^{1}$, lana awad ${ }^{1}$, Mhd Baraa Habib ${ }^{1}$, Abdul Aziz Zafar. ${ }^{2}$, and afaf albattah ${ }^{1}$

${ }^{1}$ Hamad Medical Corporation

${ }^{2}$ Hamad General Hospital

October 14, 2021

\author{
Abstract \\ Caplacizumab can be used as a treatment for TTP refractory to plasmapheresis and steroid
}

\section{Hosted file}

TTP.docx available at https://authorea.com/users/441138/articles/541705-treatment-ofrefractory-thrombotic-thrombocytopenic-purpura-with-caplacizumab-a-case-report 